\title{
Diagnóstico de la colestasis neonatal
}

\author{
Muriel Girard Florence Lacaille \\ Servicio de Gastroenterología, Hepatología y Nutrición Pediátricas, Hospital Necker-Enfants Malades, París, Francia
}

\author{
Palabras clave \\ Colestasis neonatal • Deposiciones acólicas · Atresis biliar • \\ Enfermedad metabólica
}

\section{Resumen}

La colestasis es un proceso frecuente en los recién nacidos (1 de cada 2.500 nacidos vivos) y los niños pequeños. Comprende numerosas etiologías, en ocasiones con pronósticos ominosos. En caso de colestasis neonatal, la actitud más importante consiste en observar el color de las deposiciones y excluir la atresia biliar, que debe ser tratada quirúrgicamente antes del día 45 de vida. La atresia biliar representa casi el $50 \%$ de los casos de colestasis neonatal, cuyas demás causas son numerosas. Algunos casos pueden ser tratados satisfactoriamente, como la tirosinemia de tipo I o los errores congénitos de las síntesis de ácidos biliares. No obstante, en la mayoría de los casos no existe tratamiento específico y la enfermedad evoluciona hacia la cirrosis o la insuficiencia hepática, que requiere un trasplante hepático. En la actualidad, aunque los resultados del trasplante hepático son satisfactorios, se trata de una intervención difícil con frecuentes efectos secundarios. En el futuro, el análisis y el mejor conocimiento de los mecanismos de las diferentes enfermedades colestáticas podría permitir el establecimiento de otros tratamientos como el trasplante de hepatocitos o la terapia génica, capaces de aportar nuevas perspectivas para los niños.

Copyright @ 2009 Nestec Ltd., Vevey/S. Karger AG, Basel

\section{Introducción}

La colestasis en los niños aparece frecuentemente durante las primeras semanas de vida debido a que el recién nacido se halla especialmente predispuesto por la inmadurez de la secreción biliar. La colestasis neonatal es frecuente y su incidencia es de 1 caso por cada 2.500 recién nacidos vivos. La identificación de la causa de la colestasis es muy importante si se tiene en cuenta que la más frecuente es la atresia biliar, que debe corregirse quirúrgicamente antes de los 45 días de vida [1]. Otras muchas enfermedades pueden causar colestasis en el periodo neonatal. Por otra parte, 15 enfermedades representan el 95\% de los casos y el pronóstico depende de la etiología. La colestasis es la indicación más frecuente de trasplante hepático en niños; esta intervención cambia espectacularmente el pronóstico. Actualmente se conoce un número creciente de genes participantes en enfermedades que causan colestasis neonatal; la identificación de una mutación patológica en una familia puede permitir el diagnóstico prenatal de las enfermedades más graves. Asimismo, en el futuro, la identificación de otros genes implicados en estas enfermedades permitirá un mejor conocimiento de los mecanismos de colestasis y, probablemente, nuevos tratamientos distintos del trasplante hepático.

\section{Fisiopatología de la colestasis}

La definición de colestasis se basa en manifestaciones vinculadas a un flujo de bilis disminuido o ausente o a la formación de bilis anormal. Puede deberse a alteraciones 
Fig. 1. Etapas principales de la producción de bilis en el hepatocito. La producción de bilis es un proceso activo en el que participa el transporte de ácidos biliares y otros compuestos osmóticos a través de un gradiente de concentraciones dentro del canalículo biliar. En el polo apical del hepatocito: PTAO = Proteína transportadora de aniones orgánicos; PCTS = polipéptido cotransportador de taurocolato sódico. En el polo canalicular del hepatocito: TMAOc $=$ Transportador multiespecífico de aniones orgánicos canaliculares; $\mathrm{BESB}=$ bomba exportadora de sales biliares; PRFM3 = proteína de resistencia a fármacos múltiples 3 .

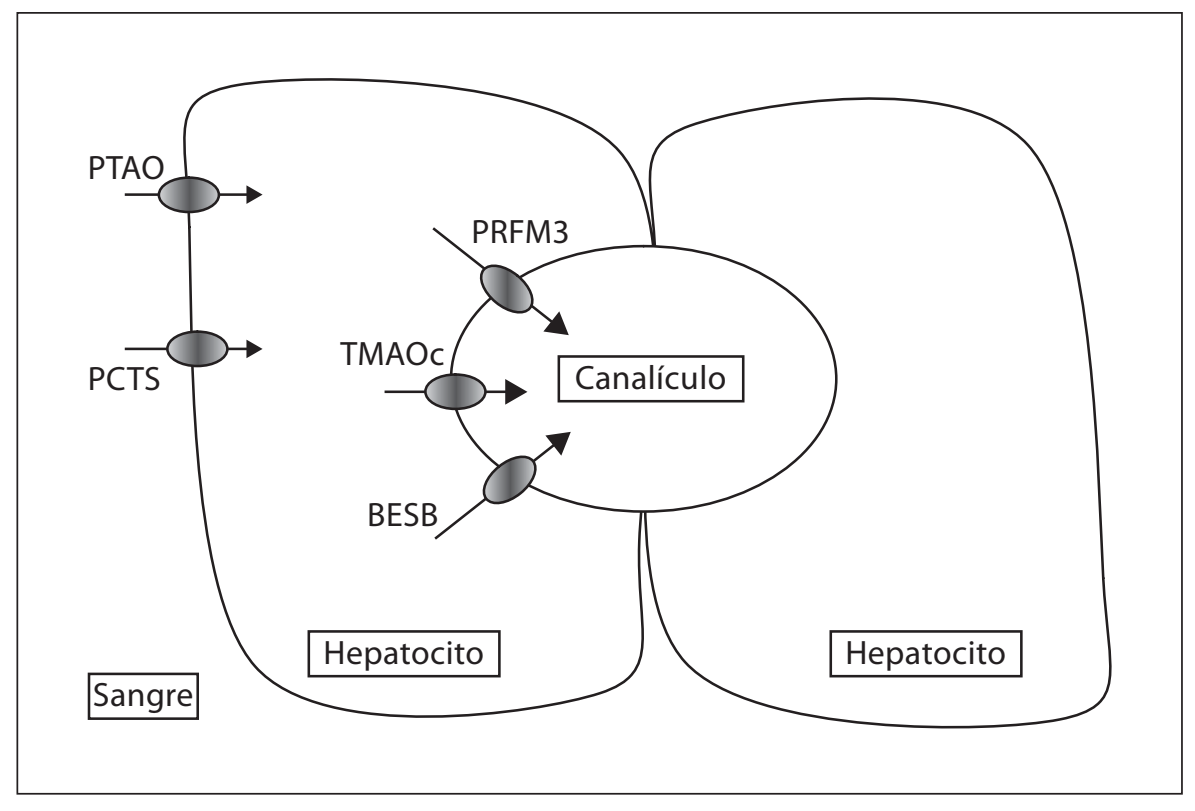

metabólicas en los hepatocitos (como consecuencia de una lesión de estas células o factores externos como infección o nutrición parenteral) o a alteraciones biliares interhepáticas o extrahepáticas.

La producción de bilis es un proceso activo en el que participa el transporte de ácidos biliares y otros compuestos osmóticos a través de un gradiente de concentraciones dentro del canalículo biliar. Esta concentración osmótica induce el movimiento pasivo de agua en el interior del canalículo. Los transportadores activos se localizan en la membrana basolateral del hepatocito para la captación de sangre sinusoidal (fig. 1). Son los siguientes: polipéptido cotransportador de taurocolato sódico (PCTS), proteína transportadora de aniones orgánicos (PTAO) y, en la membrana canalicular para la excreción biliar, bomba exportadora de sales biliares (BESB), transportador multiespecífico de aniones orgánicos canaliculares (TMAOc) y proteína de resistencia a fármacos múltiples 3 (PRFM3). La expresión de estos transportadores es modificada por hepatopatías, traumatismos o sepsis con la finalidad de proteger al hepatocito frente a los efectos citotóxicos de una elevación de la concentración de ácidos biliares. Esta regulación podría explicar probablemente el motivo por el cual la ictericia puede ser un signo precoz de sepsis. El flujo de bilis es escaso en el feto y el recién nacido debido a la inmadurez de los procesos de síntesis y transporte de ácidos biliares. En modelos animales, el PCTS y la BESB se hallan ya presentes antes del nacimiento, pero a un nivel muy bajo en comparación con los adultos. Aunque el proceso de desarrollo de los trasportadores hepatocíticos humanos no ha sido cuantificado todavía, los ácidos biliares plasmáticos no se sitúan en el intervalo normal del adulto hasta los seis meses de edad. Estas observaciones permiten suponer solidamente la predisposición del recién nacido a la colestasis $[2,3]$.

\section{Diagnóstico de la colestasis}

Debe sospecharse la presencia de colestasis si la ictericia no ha desaparecido en el curso de la segunda semana de vida. La ictericia colestática se asocia a deposiciones claras y orina oscura; durante el periodo neonatal no se presenta prurito. El hígado puede estar agrandado, firme o duro. En ocasiones se presenta esplenomegalia. La presencia de deposiciones acólicas, hígado duro e inversión visceral abdominal o torácica es muy indicativa de atresia biliar. Las características biológicas de colestasis son la hiperbilirrubinemia, principalmente conjugada, y la frecuente elevación de la $\gamma$-glutamiltransferasa, así como actividades de fosfatasa alcalina y presencia de sales biliares en el suero. Es importante distinguir entre la ictericia de la insuficiencia hepática y la ictericia colestática (en esta situación, el tiempo de protrombina se normaliza tras la inyección de vitamina $\mathrm{K}_{1}$ ). En la colestasis se presenta malabsorción de las vitaminas liposolubles y carencia de vitamina $K_{1}$, que predispone a la hemorragia; la inyección de vitamina $K_{1}$ no debe omitirse nunca. Debe 
realizarse ecografía abdominal en todos los lactantes colestáticos, a cargo de un radiólogo experimentado. Es rara la dilatación de los conductos biliares intrahepáticos, indicativa de la presencia de un cálculo biliar o un quiste coledociano. Aunque una vesícula biliar pequeña y dismórfica es muy indicativa de atresia biliar, este signo no es específico dado que la vesícula biliar está vacía cuando la colestasis es grave. Por el contrario, una vesícula biliar normal no excluye la atresia biliar teniendo en cuenta las formas quísticas de la misma. El objetivo estriba en que una atresia biliar no pase inadvertida. El procedimiento diagnóstico debe realizarse lo más rápidamente posible. Si las deposiciones son permanentemente acólicas, debe efectuarse una biopsia hepática o una colangiografía para excluir o confirmar la atresia biliar.

\section{Etiologías}

\section{Enfermedades extrahepáticas}

Las causas exclusivamente extrahepáticas de la colestasis neonatal son raras (5\%) y pueden diagnosticarse fácilmente por ecografía. Las deposiciones pueden ser permanente o transitoriamente acólicas.

La litiasis del conducto biliar común puede causar una colestasis grave y su manifestación puede consistir en deposiciones acólicas intermitentes. Los factores predisponentes son la hiperhemólisis o la nutrición parenteral. La eliminación espontánea del cálculo es frecuente. Por el contrario, una colecistografía percutánea puede permitir la eliminación del cálculo. En principio, no se producen recidivas.

La perforación espontánea del conducto biliar es muy rara y aparece a menudo en la unión del conducto biliar común y el conducto cístico. La presentación clínica consiste en peritonitis biliar, acumulación de bilis o estenosis biliar. El tratamiento es quirúrgico.

Un quiste coledociano se descubre en raras ocasiones durante el periodo neonatal, si bien es la segunda causa quirúrgica más frecuente de la colestasis neonatal. En el periodo neonatal, la presentación clínica es habitualmente similar a la de la atresia biliar, pero puede consistir también en un inicio súbito de colestasis o colangitis con dolor abdominal y pancreatitis. El diagnóstico se establece por ecografía o tomodensitometría. El tratamiento es quirúrgico con ablación total del quiste residual para evitar una transformación maligna ulterior. El pronóstico a largo plazo es muy favorable, con función hepática normal.

Diagnóstico de la colestasis neonatal

\section{Causas extrahepáticas e intrahepáticas}

\section{Atresia biliar}

La atresia biliar es la causa más frecuente de colestasis neonatal y representa casi el 50\% de los casos. Es la primera indicación de trasplante hepático en niños. La incidencia es de 1 caso por cada 10.000 recién nacidos vivos. Resulta de una obliteración del conducto biliar que se inicia en el útero o en el periodo postnatal muy precoz. La alteración del conducto biliar (destrucción inflamatoria) afecta a todo el árbol biliar en el $80 \%$ de los casos. Aunque la causa de atresia biliar sigue siendo desconocida, en algunos casos puede sospecharse un origen genético debido a la asociación de algunos elementos heterotáxicos, como inversión visceral abdominal o torácica, con poliesplenia en alrededor del $20 \%$ de los casos y una incidencia elevada (6 veces superior) en algunas poblaciones [4]. El objetivo de la cirugía es restablecer el flujo de bilis a través de un asa intestinal (intervención de Kasai). La intervención será satisfactoria si los conductos biliares intrahepáticos en el hilio no están todavía ocluidos. Dado que la destrucción inflamatoria parece progresiva, la intervención de Kasai debe efectuarse lo más pronto posible. Antes de los 45 días de vida, el niño tiene un $80 \%$ de probabilidades de permanecer libre de ictericia a los 3 años de edad. Si la intervención de Kasai se practica después de los 45 días de vida, el éxito de la intervención disminuye espectacularmente. Si la operación de Kasai fracasa, la enfermedad evoluciona hacia la cirrosis biliar, en cuyo caso debe efectuarse un trasplante hepático entre el primer y el segundo año de vida. Incluso cuando la intervención de Kasai es eficiente, en una gran mayoría de casos la cirrosis está ya presente, con riesgo de hipertensión portal y hemorragia intestinal. El niño puede presentar también colangitis, ictericia e insuficiencia hepática crónica, así como fallo hepático agudo; un trasplante hepático es a menudo necesario en una etapa posterior. Sin trasplante hepático, sólo el 10\% de los niños permanecen con vida a los 20 años [5]. Debido a la gravedad y a la importancia del diagnóstico precoz, debe sospecharse sistemáticamente la presencia de una atresia biliar en casos de colestasis neonatal. La sospecha diagnóstica es alta si el niño presenta permanentemente deposiciones acólicas (especialmente a partir del nacimiento) con hepatomegalia dura, si existen elementos heterotáxicos, si se observa un quiste biliar en el hilio y si la vesícula biliar es pequeña y dismórfica después del ayuno. Aunque la ecografía puede contribuir al diagnóstico, éste se establece realmente por colangiografía practicada bien sea en el momento de una laparosco-

Ann Nestlé [Esp] 2008;66:109-120 
pia o directamente en la laparotomía, ambos procedimientos seguidos de la intervención de Kasai si procediera.

\section{Colangitis esclerosante neonatal}

La colangitis esclerosante neonatal es una enfermedad muy rara, diferente de la colangitis esclerosante primaria que se observa en niños mayores asociada a enfermedad intestinal inflamatoria. La presentación clínica consiste en colestasis neonatal grave con deposiciones acólicas, que puede remedar la atresia biliar. El diagnóstico se establece por colangiografía, que muestra conductos intrahepáticos permeables, si bien un estrechamiento irregular de los conductos biliares extrahepáticos o intrahepáticos. Habitualmente, no se detecta enfermedad intestinal en estos pacientes. Frecuentemente se observa una resolución de la ictericia, aunque con cirrosis e hipertensión portal precoces; pocos niños necesitan un trasplante hepático [6]. Algunas observaciones permiten suponer un origen genético debido a la existencia de casos familiares [7]. Unos pocos casos asociados a ictiosis están ligados a una mutación del gen Claudin-1, que codifica una molécula de unión intercelular hermética [8]. Se han descrito otros casos de este síndrome de ictiosis-colangitis esclerosante neonatal (ICEN), que muestran una expresión clínica variable en el fenotipo cutáneo y hepático [9]. No obstante, la mayoría de niños con colangitis esclerosante neonatal no presentan ictiosis, lo que da a entender que la enfermedad es heterogénea.

\section{Colangitis esclerosante con inicio postnatal}

La colangitis puede asociarse a otras enfermedades, como histiocitosis $\mathrm{X}$, síndrome de inmunodeficiencia, enfermedad intestinal inflamatoria crónica o hepatitis autoinmune y psoriasis congénita. No obstante, en algunos casos, no se detecta ninguna enfermedad asociada [6].

\section{Causas intrahepáticas}

La colestasis intrahepática representa casi el $50 \%$ de las causas de colestasis neonatal y comprende un grupo heterogéneo de enfermedades.

\section{Enfermedades infecciosas}

Las causas clásicas son fetopatías (infecciones de rubéola congénita, toxoplasmosis, citomegalovirus y sífilis), frecuentemente asociadas a bajo peso al nacer y síntomas neurológicos o hematológicos. Las infecciones bacterianas (la más frecuente es la infección de las vías urinarias por Escherichia coli) pueden inducir la inhibición del transporte canalicular de ácidos biliares y causar colestasis.

\section{Causas tóxicas}

El agente tóxico más frecuente para el hígado durante el periodo neonatal es la nutrición parenteral. Los mecanismos son probablemente complejos, con asociación de infecciones recurrentes, respuesta inflamatoria, contenido inapropiado de la nutrición parenteral, ausencia de circulación enterohepática e inmadurez del transporte biliar [10]. En algunos casos, los niños con colestasis muy grave inducida por la nutrición parenteral pueden presentar insuficiencia hepática. La lesión farmacológica puede ser un factor adicional de las alteraciones hepáticas. Un polimorfismo asociado a una disminución de la expresión de la $B E S B$ hepática fue significativamente más frecuente en la colestasis inducida por fármacos en pacientes adultos [11], lo que permite suponer el papel que, en los recién nacidos, podrían desempeñar también factores genéticos en la inducción de la hepatopatía por nutrición parenteral.

\section{Isquemia}

La isquemia en recién nacidos se acompaña frecuentemente de colestasis, sin síntomas distintos de la colestasis no complicada. La colestasis se asocia a varios factores contributivos relacionados con la gravedad del trastorno neonatal. Los niños nacidos antes de 35 semanas de edad presentan un mayor riesgo (tres veces superior) de colestasis neonatal $[12,13]$.

\section{Enfermedades genéticas}

Síndrome de Alagille

El síndrome de Alagille es un trastorno dominante autosómico que recibe también el nombre de 'hipoplasia sindrómica de los conductos biliares'. Afecta a todos los grupos étnicos. Se caracteriza por ictericia neonatal, colestasis intrahepática y trastornos del desarrollo, que afectan al hígado, el corazón, las vértebras, los ojos y la cara. El síndrome de Alagille representa del 10 al 15\% de la colestasis neonatal y su frecuencia es de 1 caso por cada 100.000 recién nacidos vivos. El diagnóstico clínico se basa en la asociación de dismorfia facial (frente prominente, ojos hundidos, hipertelorismo y mentón puntiagudo pequeño; fig. 2) con embriotoxon posterior, vértebras en forma de mariposa, estenosis periférica de la arteria pulmonar (u otra cardiopatía) y colestasis debida a la escasez de conductos biliares interlobulares. La herencia es 
dominante, con penetrancia variable y una gran heterogeneidad en las manifestaciones clínicas, que permite suponer la importancia de varios factores en la patogénesis. El pronóstico es peor en niños que presentan ictericia colestática neonatal, cuya resolución es infrecuente. Una mayoría de ellos necesitarán trasplante hepático en la infancia [14]. No obstante, las complicaciones hepáticas graves son también posibles en la hepatopatía de inicio tardío, que exige un seguimiento durante toda la vida. El diagnóstico molecular es posible: el $70 \%$ de los pacientes presentan una mutación en el gen jagged-1 que codifica un receptor Notch que participa en la diferenciación y el desarrollo celulares. El mecanismo consiste probablemente en una haploinsuficiencia. No existe una correlación genotipo-fenotipo. No se observan agrupamientos y la mayoría de las mutaciones son de tipo específico familiar. Los casos esporádicos son los más frecuentes (70\%) en relación con la elevada tasa de mutaciones de novo [15]. Se registra una tasa elevada de mutaciones nuevas, que dificulta el diagnóstico molecular.

\section{Deficiencia de antitripsina $\alpha_{1}$}

La deficiencia de antitripsina $\alpha_{1}$ es un trastorno recesivo corriente, que afecta a 1 caso de cada 2.000 nacidos vivos. El diagnóstico se sustenta en una electroforesis proteínica con un pico ausente de globulinas $\alpha$ y se confirma por la determinación específica de antitripsina $\alpha_{1}$ en el suero y el análisis de proteínas fenotípicas. La proteína antitripsina $\alpha_{1}$ es sintetizada en el hígado y exportada al pulmón, en cuyo órgano actúa como una antiproteasa, evitando la lesión alveolar causada por la elastasa leucocítica. La proteína anormal posee un fenotipo 'Z', no puede ser exportada fuera del hígado y polimeriza en el retículo endoplasmático. La acumulación intrahepática de la proteína $Z$ mutante en el hígado induce la activación de autofagia, lesión de las mitocondrias y activación de la caspasa, con el consiguiente daño hepatocelular. Sólo los pacientes con genotipo ZZ presentan colestasis en la infancia o neumopatía y hepatopatía en la edad adulta. La hepatopatía puede aparecer en cualquier edad. La mayoría de los niños están libres de disfunción hepática significativa y sólo una pequeña proporción de niños $\mathrm{ZZ}$ homocigóticos (10 a 15\%) presentan colestasis durante el periodo neonatal. La variable presentación clínica permite suponer una contribución importante por parte de modificadores genéticos y ambientales. Aunque el estado de portador heterocigótico para el gen $\mathrm{Z}$ mutante, presente en el 1,5 al 3\% de la población, no es causa de lesión hepática, puede constituir un gen modificador para otras hepatopatías [16-18]. La característica clínica de la pre-

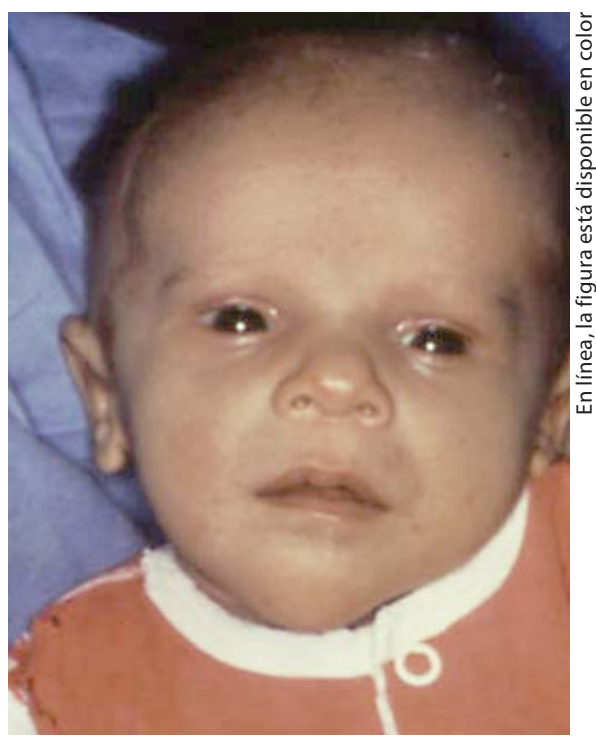

Fig. 2. Fotografía de un lactante con síndrome de Alagille. Adviértanse los rasgos característicos: frente prominente, ojos hundidos, hipertelorismo y mentón puntiagudo pequeño.

sentación neonatal puede ser muy similar a la de la atresia biliar. No se dispone de tratamiento específico y el desenlace es variable: en el 50\% de los niños desaparece la anomalía hepática en la edad adulta, el 10\% seguirán siendo colestáticos y necesitarán un trasplante hepático y algunos de los niños (40 a 50\%) presentarán una hepatopatía compensada con alteraciones leves y la posibilidad de necesitar un trasplante hepático en la edad adulta.

Fibrosis quística

La fibrosis quística es una de las enfermedades hereditarias más frecuentes en los de la raza blanca (1 caso de cada 2.000 nacidos vivos en Europa). La presentación clínica puede ser variable. La colestasis neonatal es una manifestación clínica primaria característica, pero rara, en la fibrosis quística, y lo más frecuente es que se asocie a un íleo meconial. El mecanismo consiste probablemente en la obstrucción de los conductillos biliares por secreciones mucoides anormales. Los pacientes pueden presentar una colestasis completa que imita la atresia biliar. En pacientes con fibrosis quística y colestasis neonatal, $1 / 3$ presentan otra condición, lo que incrementa el riesgo de colestasis, como deficiencia de antitripsina $\alpha_{1}$, hipopituitarismo, asfixia perinatal y nutrición parenteral total [19]. 
Colestasis intrahepática familiar progresiva

La colestasis intrahepática familiar progresiva (CIFP) es un grupo heterogéneo de enfermedades que representa el $10 \%$ de colestasis neonatal. La presentación clínica consiste en colestasis neonatal o infantil con prurito. Evoluciona hacia la cirrosis o la insuficiencia hepática en la infancia, que a menudo obliga a realizar un trasplante hepático. La herencia es autonómica recesiva. Recientemente se han identificado tres grupos de enfermedades con características fenotípicas particulares (ver el capítulo siguiente).

La CIFP-1 y la CIFP-2 se caracterizan por colestasis precoz con insuficiencia hepática frecuente y prurito intenso; la actividad de la gamma GT es siempre normal. La CIFP-1 se debe a una mutación en el gen FICI, que codifica una ATPasa presente en el polo canalicular del hepatocito, que desempeña un papel todavía desconocido en la formación de bilis. Algunos pacientes pueden experimentar un deterioro de otros órganos, excepto el hígado, como diarrea, insuficiencia renal, pancreatitis y sordera. La CIFP-2 se debe a una mutación en el gen $B E S B$, que participa en la exportación de sales biliares en los canalículos. La evolución es a menudo más grave que en la deficiencia de CIFP-1.

La CIFP-3, debida a una mutación en el gen PRFM3, es un transportador transmembránico de fosfolípidos hepatocíticos. En comparación con los dos primeros procesos, la deficiencia de CIFP-3 se caracteriza por menos prurito, hipertensión portal frecuente, insuficiencia hepática tardía y actividad de gamma GT elevada.

La mitad de los pacientes con CIFP responden bien al ácido ursodesoxicólico. Algunos niños pueden beneficiarse de un tratamiento de derivación biliar externa parcial (exceptuando los afectados de deficiencia de PRFM3). Los niños que respondan sólo parcialmente o los que no respondan necesitarán un trasplante hepático [20-22]. Si se dispone de un diagnóstico molecular para la familia, en algunos laboratorios es posible establecer un diagnóstico prenatal [23].

Errores congénitos de la síntesis de ácidos biliares

Los errores congénitos de la síntesis de ácidos biliares son trastornos específicos de los enzimas que participan en las reacciones que convierten el colesterol en los ácidos cólico y quenodesoxicólico [24]. En el nacimiento o en la primera infancia, los sujetos afectados presentan ictericia colestática, carencia de vitaminas liposolubles y deposiciones acólicas o grasas (esteatorrea). Las transaminasas séricas suelen estar elevadas, la actividad gamma GT es normal y a menudo está presente una hiperbilirrubine- mia conjugada. Aunque las biopsias hepáticas pueden revelar alteraciones inespecíficas, como transformación de los hepatocitos en células gigantes, inflamación y fibrosis, la actividad gamma GT es normal y la colestasis canalicular y hepatocítica es habitual. La identificación de trastornos de la síntesis de ácidos biliares se basa en el análisis por espectrometría de masa de la orina y el suero para establecer la ausencia o la reducción notable de determinados ácidos biliares primarios, los ácidos cólico y quenodesoxicólico, al mismo tiempo que la presencia de excesivas cantidades de ácidos biliares y esteroles atípicos, sintetizados como consecuencia de la deficiencia enzimática.

Hasta la fecha se han identificado mutaciones de varios genes (fig. 3) $[25,26]$ :

Deficiencia de $3 \beta$-hidroxi-C27-esteroide deshidrogenasa oxidorreductasa (HSD3B7); deficiencia de $\Delta 4-3$ oxosteroide $5 \beta$-reductasa (AKR1D1); deficiencia de oxisterol $7 \alpha$-hidroxilasa (CYP7B1); deficiencia de 2-metilacil-CoA racemasa; deficiencia de esterol 27-hidroxilasa (CYP27A1); deficiencia de ácido biliar-CoA:aminoácido $\mathrm{N}$-aciltransferasa (BAAT); deficiencia de colesterol $7 \alpha$ hidroxilasa (CYP7A1).

El diagnóstico precoz es importante porque los pacientes pueden ser tratados satisfactoriamente mediante la administración oral de ácido cólico. La normalización de las enzimas hepáticas y la bilirrubina en suero, así como la resolución de la lesión histológica, son respuestas coherentes al tratamiento con ácidos biliares, y la necesidad de trasplante hepático puede evitarse en la mayoría de los casos. Los trastornos biosintéticos de los ácidos biliares, en particular la deficiencia de 3 $\beta$-hidroxi-C27-esteroide deshidrogenasa oxidorreductasa, puede causar también colestasis crónica de inicio tardío; en estos sujetos, la historia clínica suele revelar una pauta de transaminasas ligeramente elevadas en la lactancia que, a menudo, se resuelve, sólo para reaparecer posteriormente con raquitismo por deficiencia de vitamina $\mathrm{D}$ de inicio precoz.

\section{Enfermedad de Niemann-Pick de tipo C}

La enfermedad de Niemann-Pick de tipo $\mathrm{C}$ es un raro trastorno autosómico recesivo de almacenamiento de lípidos, que se caracteriza por neurodegeneración progresiva. La presentación clínica es heterogénea y la edad de inicio varía entre el periodo perinatal y la edad adulta. En el recién nacido se presentan frecuentemente hepatomegalia y esplenomegalia, asociadas a ictericia colestática prolongada. Aunque lo habitual es que la ictericia desaparezca espontáneamente, en ocasiones aparece un fallo hepático rápidamente letal. La edad en el momento del 


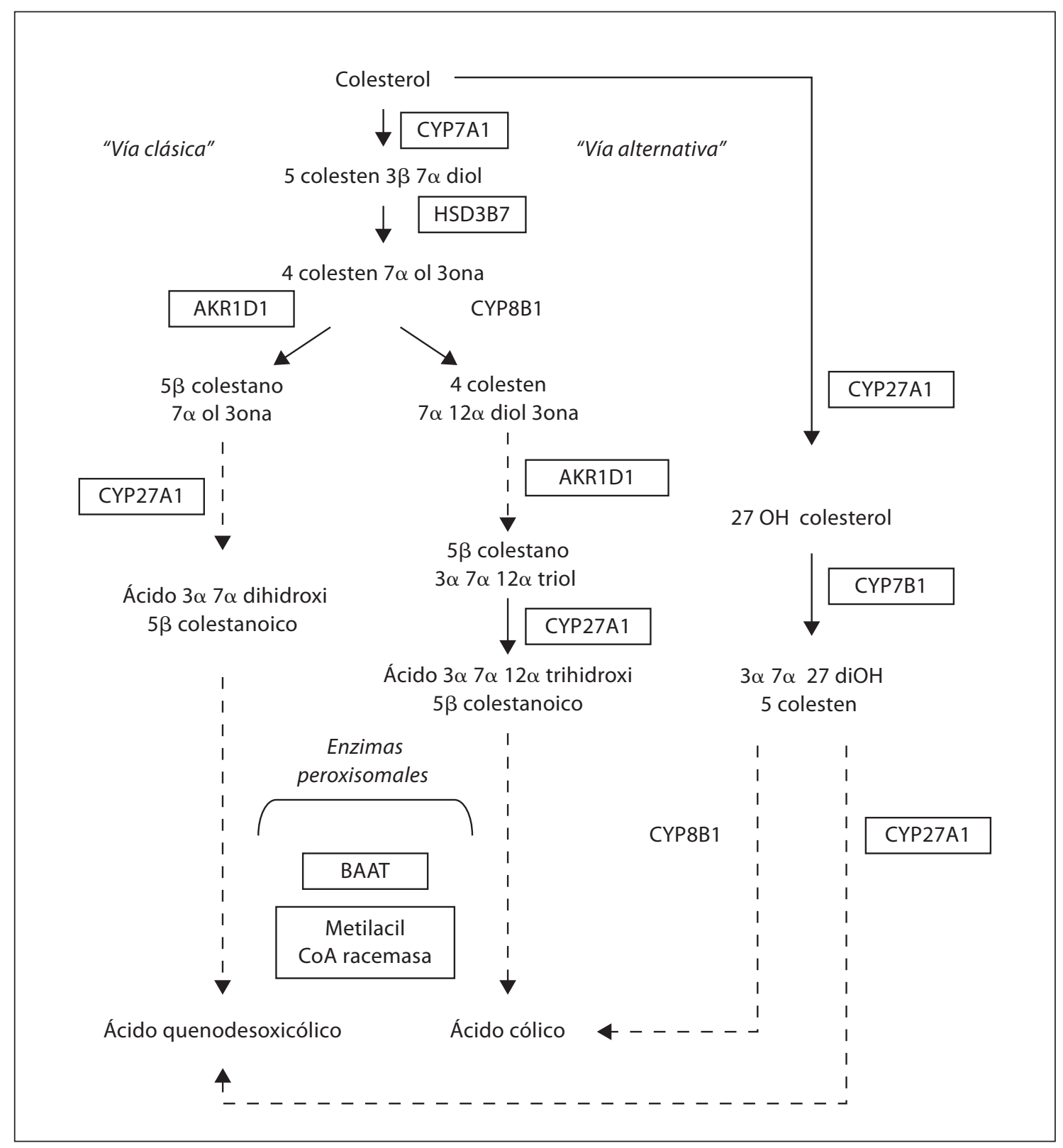

Fig. 3. Vías biosintéticas de ácidos biliares. La vía clásica de biosíntesis de los ácidos biliares está presente sólo en los hepatocitos. Sin embargo, la vía alternativa existe en todos los tejidos. Sólo se muestran las fases y enzimas reguladoras principales. La vía clásica sintetiza dos ácidos biliares primarios, el ácido cólico y el ácido quenodesoxicólico. Las enzimas encuadradas son las conocidas por participar en los errores congénitos de la síntesis de ácidos

inicio de los síntomas neurológicos y su evolución determinan la gravedad del proceso. La enfermedad de Niemann-Pick se diagnostica en el 8\% de los lactantes que se evalúan por colestasis; debe considerarse en todos los lactantes con colestasis, sobre todo en los que presentan es- biliares. CYP7A1 = Colesterol 7 $\alpha$ - hidroxilasa; HSD3B7 $=3 \beta$ hidroxil $\Delta 5$-C27-esteroide oxidorreductasa; CYP8B1 = esterol $12 \alpha$-hidroxilasa; AKR1D1 $=\Delta 4$-3-oxosteroide $5 \beta$-reductasa; CYP27A1 = esterol 27-hidroxilasa; CYP7B1 $=$ oxisterol $7 \alpha$-hidroxilasa; $\mathrm{BAAT}=$ ácido biliar-CoA:aminoácido N-aciltransferasa. plenomegalia o síntomas neurológicos asociados. Se sospecha el diagnóstico cuando se detectan células espumosas cargadas de lípidos en la médula ósea y el hígado. El gen mutado participa en el transporte intracelular de colesterol y causa la acumulación de colesterol no esterifi- 
cado en los lisosomas. El diagnóstico se establece demostrando estas anomalías en fibroblastos cultivados. Cabe la posibilidad de establecer también un diagnóstico molecular. Participan dos genes, NPC1 y NPC2, que permiten un diagnóstico prenatal [27]. No se dispone de ningún tratamiento específico.

\section{Trastornos de las mitocondrias}

Los trastornos de las mitocondrias pueden manifestarse como fallo hepático agudo neonatal, esteatohepatitis, colestasis o cirrosis con insuficiencia hepática crónica de inicio insidioso. Habitualmente, se presentan síntomas neuromusculares significativos, afectación multisistémica y acidemia láctica. La hepatopatía suele ser progresiva y por último letal. Con la posibilidad del diagnóstico genético y prenatal, en los últimos años se han identificado varios trastornos moleculares (mutaciones en genes nucleares, como SCO1, BCS1L, POLG, DGUOK y MPV17 y eliminación o reordenación del ADN mitocóndrico). El tratamiento médico actual de las hepatopatías mitocóndricas es en gran medida ineficaz y el pronóstico es habitualmente ominoso. El papel del trasplante hepático en pacientes con insuficiencia hepática continua siendo mal definido por la naturaleza sistémica de la enfermedad, con posibilidad de afectación de otros órganos que el hígado $[28,29]$.

\section{Galactosemia}

La galactosemia se caracteriza por una deficiencia del metabolismo de la galactosa con resultado de una acumulación de galactosa-1-fosfato. Es un trastorno autosómico recesivo y afecta a 1 caso de cada 35.000 recién nacidos en Europa. Los síntomas clínicos aparecen durante los primeros días de vida y entre ellos destacan vómito, ictericia, letargia, hepatomegalia e insuficiencia hepática en un bebé amamantado o alimentado con una fórmula láctea que contiene lactosa. Si se deja sin tratamiento, el proceso evoluciona rápidamente hacia una insuficiencia hepática y renal con sepsis causada por bacterias gramnegativas. Después de varios días o semanas aparecen cataratas que se tornan rápidamente irreversibles. El diagnóstico se basa en la detección de la acumulación de galactosa-1-fosfato en los eritrocitos (prueba de spot), medición de la actividad de la galactosa-1-fosfato uridiltransferasa en los eritrocitos, determinación de una deficiencia en una de las enzimas de la via metabólica e identificación de la mutación génica. En la vía metabólica de la galactosa participan los tres enzimas siguientes: (1) galactosa-1-fosfato uridiltransferasa (el más corriente); (2) galactocinasa; (3) uridin difosfato galactosa-4-epimerasa.
El diagnóstico precoz es importante con objeto de iniciar una dieta exenta de lactosa. La insuficiencia hepática desaparece en el curso de unos pocos días. La dieta libre de lactosa tiene que mantenerse por toda la vida.

En caso contrario, aparecen durante la infancia complicaciones neurológicas (disminución de los rendimientos intelectuales con la edad) e hipogonadismo hipergonadotrópico (disfunción ovárica). A pesar de una dieta estricta, las complicaciones a largo plazo, como retraso mental, dispraxia verbal, anomalías motoras e hipogonadismos, son frecuentes. Se ha sugerido que estas complicaciones pueden ser el resultado de la síntesis endógena de galactosa o de una galactosilación anormal. Nuevas estrategias terapéuticas destinadas a la prevención de la producción de galactosa-1-fosfato deben desarrollarse [30].

Tirosinemia de tipo I

La tirosinemia de tipo I es un trastorno autosómico recesivo debido a la deficiencia del fumarilacetoacetato, una enzima implicada en el catabolismo de la tirosina. En la forma neonatal aguda, el inicio se produce entre 2 y 6 semanas de edad, con síntomas de insuficiencia hepatocelular entre los que destacan vómito, diarrea, ictericia, hipoglucemia, edema, ascitis y hemorragia.

La septicemia es una complicación frecuente. El diagnóstico se confirma por la detección de ácido $\delta$-aminolevulínico y succinilacetona en la orina y por la medición de la actividad enzimática en los fibroblastos. El tratamiento se basa en una dieta con restricción de tirosina y la administración de NTBC [(2-nitro-4-trifluorometilbenzoil)-ciclohexano-1,3-diona], que inhibe la tirosinoxidasa y evita la acumulación de la succinilacetona tóxica [31]. A pesar de este tratamiento, algunos pacientes presentan hepatoma con elevación de $\alpha$-fetoproteína y pasan a ser candidatos de un trasplante hepático [32]. El diagnóstico prenatal se basa en el análisis de los metabolitos, el estudio enzimático o la detección de la mutación cuando es conocida.

\section{Trastornos peroxisomales}

Numerosos trastornos peroxisomales ejercen un impacto sobre la síntesis de ácidos biliares [33]. El síndrome de Zellweger, que se define por un número reducido de peroxisomas, se caracteriza por rasgos craneofaciales dismórficos, hipotonía intensa, convulsiones, así como disfunciones hepáticas y renales. Se observan hepatomegalia y colestasis con fibrosis progresiva. El diagnóstico bioquímico se basa en el estudio de los ácidos grasos de cadena muy larga. Este deterioro metabólico resulta en la 
Fig. 4. Algoritmo en el caso de deposiciones muy claras o acólicas. El primer paso consiste en saber si las vías biliares están dilatadas o no. En ausencia de obstrucción extrahepática, deben excluirse el síndrome de Alagille, la fibrosis quística y la deficiencia de antitripsina $\alpha_{1}$. Si no se descubre una etiología evidente, debe realizarse una biopsia hepática o una colangiografía.

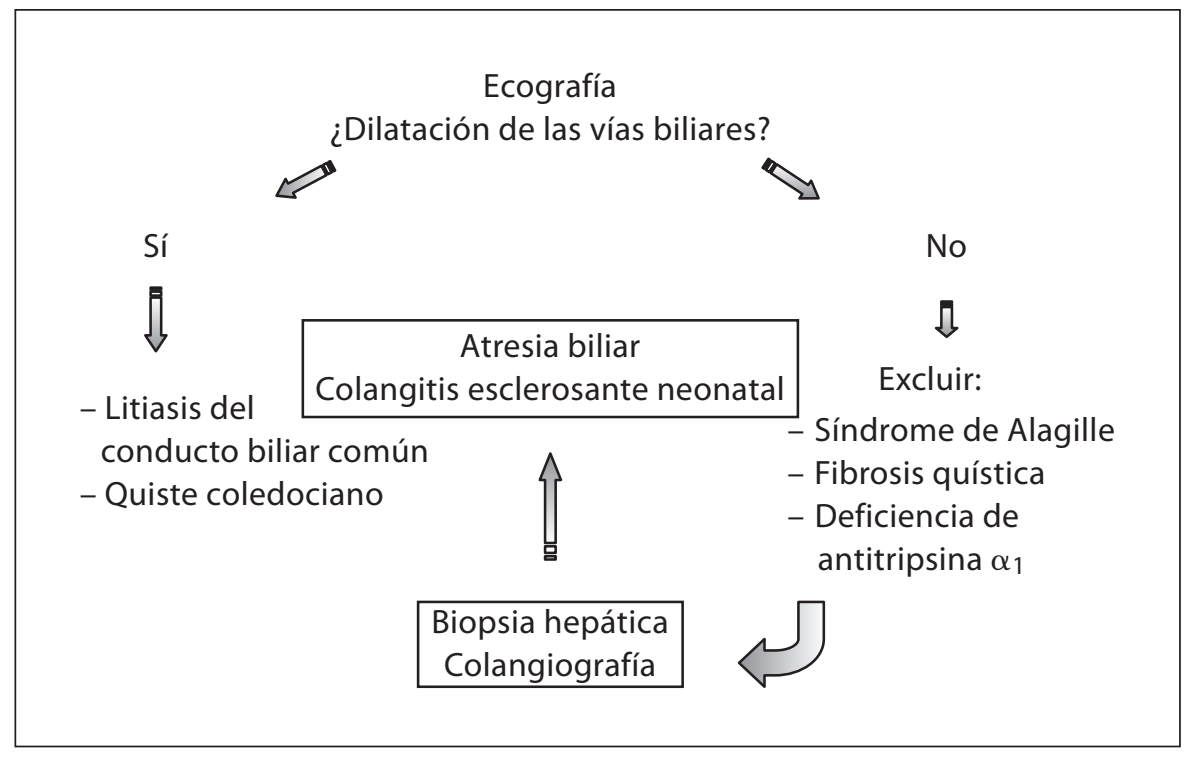

acumulación de metabolitos tóxicos y daña a las células en fase de desarrollo. La enfermedad se transmite como rasgo autosómico recesivo. La mayoría de los lactantes afectados por el síndrome de Zellweger fallecen en el curso del primer año de vida como consecuencia de apnea o neumopatía en relación con una infección o una convulsión intratable.

Síndromes de glucoproteínas deficientes de hidratos de carbono

Los síndromes de glucoproteínas deficientes de hidratos de carbono (GDHC) son un grupo de trastornos de la síntesis de las glucoproteínas caracterizados por afectación multivisceral. El síndrome de GDHC de tipo Ib se caracteriza por manifestaciones hepáticas e intestinales (diarrea, vómito, hepatomegalia con fibrosis hepática) y la ausencia de afectación neurológica. La fibrosis hepática puede evolucionar hacia la cirrosis y la insuficiencia hepática [34]. Esta forma de síndrome de GDHC puede ser tratada satisfactoriamente con manosa por vía oral. El diagnóstico biológico se basa en la demostración de la glucosilación anormal de las glucopoteínas séricas, la medición de las actividades de los enzimas leucocíticos y mutaciones en los genes correspondientes. Existe una posibilidad de diagnóstico prenatal del síndrome de GDHC.

\section{Colestasis neonatal transitoria}

La colestasis neonatal transitoria representa del 5 al $10 \%$ de las colestasis neonatales. A menudo recibe el nom- bre de 'hepatitis neonatal', si bien no se ha detectado ningún agente infectivo. La evolución es siempre favorable hacia la resolución. El diagnóstico debe establecerse con cautela después de un seguimiento cuidadoso. Cuando se realiza una biopsia hepática se observa una hepatitis de células gigantes multinucleadas, con leve proliferación de los conductillos y fibrosis inflamatoria portal moderada [35]. El mecanismo es probablemente multifactorial: inmadurez de la secreción de ácidos biliares en el periodo neonatal, lesión fetal hepática (isquemia, infección), ayuno prolongado (nutrición parenteral) y posiblemente, factores genéticos que predisponen a la colestasis [36]. Antes de establecer este diagnóstico es necesario excluir las demás causas de colestasis neonatal.

\section{Abordaje práctico del diagnóstico de colestasis neonatal}

En casos de sospecha de colestasis, el dato principal y más importante en la evaluación es el color de las deposiciones. Si las deposiciones son acólicas, la atresia biliar debe ser diagnosticada o excluida. Si se excluye una patología obstructiva del árbol biliar extrahepático, el diagnóstico de atresia biliar es probable. Deben efectuarse una colangiografía y una biopsia hepática. Si la vía biliar no es permeable debe practicarse una intervención de Kasai. Por el contrario, si la vía biliar es permeable debe considerarse otro diagnóstico y una posible colangitis esclerosante neonatal (fig. 4). 
Fig. 5. Algoritmo en el caso de deposiciones claras pero no acólicas. Debe descartarse la presencia de un obstáculo extrahepático. En casos de nivel bajo de gamma GT son posibles una CIFP-1 o CIFP-2 y errores congénitos de la síntesis de bilis. En casos de nivel elevado de gamma GT debe excluirse el síndrome de Alagille, la fibrosis quística y la deficiencia de antitripsina $\alpha_{1}$. Si ninguna etiología es evidente debe realizarse una biopsia hepática y una colangiografía.

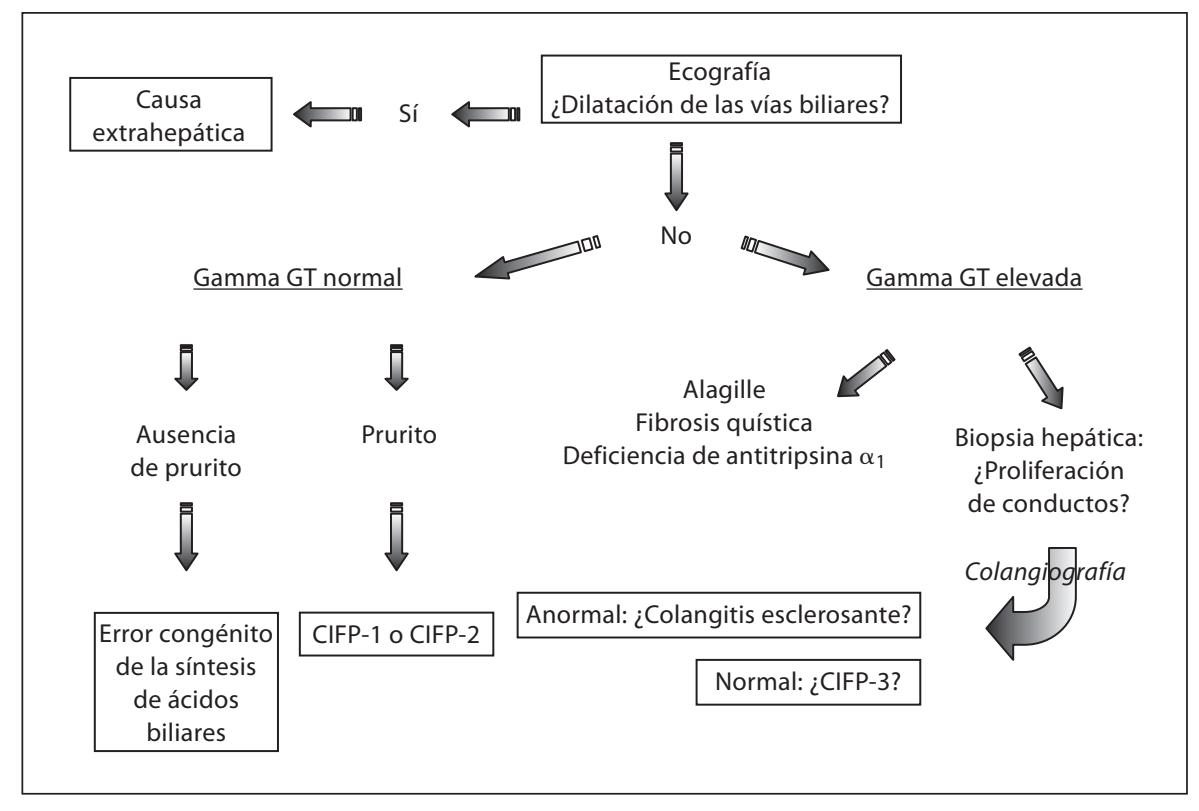

\section{Atresia biliar}

Con objeto de no pasar por alto la atresia biliar debe considerarse lo siguiente: (1) La colestasis neonatal es una atresia biliar hasta que no se demuestre definitivamente lo contrario; (2) el diagnóstico de atresia biliar es una urgencia; (3) no debe pensarse primero en la ictericia por lactancia materna; (4) preguntar a los padres el color de las deposiciones; (5) no debe suponerse que un crecimiento normal excluye una hepatopatía grave; (6) no deben suspenderse las exploraciones después de detectar citomegalovirus en la orina; (7) no debe pensarse que la bilirrubinemia es siempre muy elevada en la atresia biliar; (8) efectuar una biopsia hepática si el diagnóstico es ambiguo; (9) organizar una colangiografía operatoria cuando no se ha excluido la atresia biliar y transcurre el tiempo.

Si las deposiciones son claras pero no acólicas, las pruebas bioquímicas (relacionadas con las diversas causas de colestasis neonatal) o una biopsia hepática contribuirán al diagnóstico. Son importantes parámetros diferentes, como la dilatación de las vías biliares y el nivel de la actividad gamma GT (fig. 5).

\section{Tratamiento de un niño colestático}

Aparte de un posible tratamiento específico, el tratamiento de las consecuencias de la colestasis es importante para evitar complicaciones. La consecuencia principal es el deterioro de la absorción de lípidos debido a la au- sencia de bilis en el intestino. La malabsorción de vitaminas liposolubles puede resultar en hemorragia (deficiencia de vitamina $K$ ), raquitismo (deficiencia de vitamina D), neuropatía (deficiencia de vitamina E) y enfermedad ocular (deficiencia de vitamina A). Las vitaminas liposolubles deben administrarse por vía oral a dosis elevadas, siempre que la colestasis no sea demasiado grave y el nivel de vitaminas A, E y D se compruebe con regularidad. Si la colestasis es grave, o en caso de baja respuesta a la vía oral, las inyecciones parenterales son imperativas.

El deterioro de la absorción de los lípidos y la colestasis pueden perjudicar gravemente el crecimiento. Las fórmulas para lactantes enriquecidas con triglicéridos de cadena media pueden mejorar la malabsorción de grasas y la nutrición. En los lactantes colestáticos, la ingestión de 180 a 200 calorías/kg/día puede ser necesaria y podría utilizarse la nutrición enteral para mantener el crecimiento.

La administración de ácido ursodesoxicólico (un ácido biliar hidrofílico) es beneficiosa en la colestasis. Sus mecanismos precisos de acción no se conocen en todos sus extremos; se produce probablemente un enriquecimiento de la reserva de ácidos biliares con ácidos biliares más hidrofílicos y la estimulación del flujo de bilis. Mejora los parámetros bioquímicos y controla la progresión de la fibrosis en la CIFP. Aunque su eficiencia no ha sido demostrada en otros síndromes colestáticos, el ácido ursodesoxicólico parece mejorar los parámetros bioquímicos y acelerar la eliminación de la ictericia en la colestasis 
Fig. 6. Consecuencias de la colestasis. En casos de colestasis, la disminución del flujo biliar es responsable de un incremento de las sales biliares en el suero, lo que causa prurito y lesión hepática. La ausencia de bilis en el intestino da lugar a malabsorción de lípidos y menoscabo del crecimiento; la malabsorción de vitaminas liposolubles causa hemorragia (deficiencia de vitamina $\mathrm{K}$ ), raquitismo (deficiencia de vitamina $\mathrm{D}$ ), neuropatía (deficiencia carencia de vitamina E) y enfermedad ocular (deficiencia carencia de vitamina A).

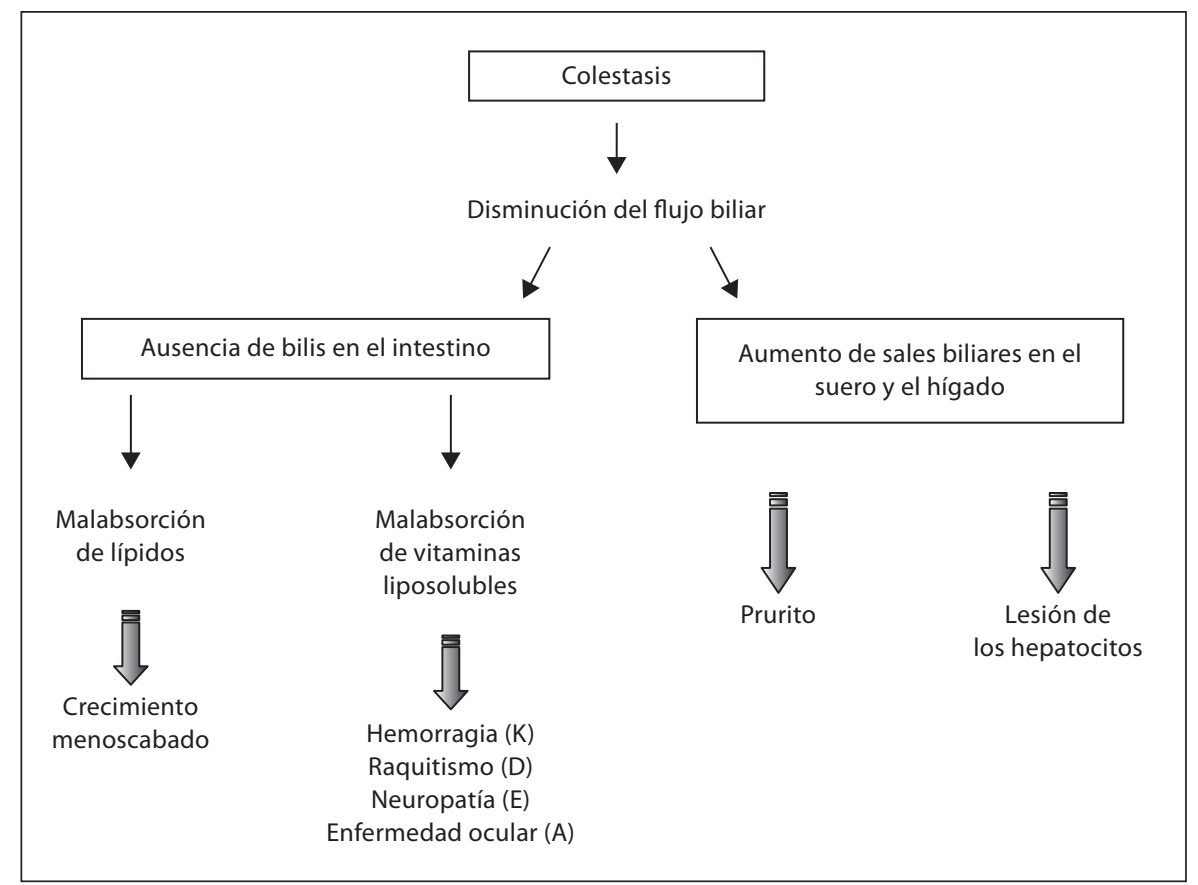

neonatal transitoria. Su único efecto secundario es la diarrea, que responde a la reducción de la dosis. Se recomienda su administración a una dosis de $20 \mathrm{mg} / \mathrm{kg} /$ día.

El prurito es un síntoma debilitante en la colestasis grave, que se hace evidente únicamente a partir del segundo semestre de vida. El tratamiento más eficaz es con rifampicina a la dosis de 5 a $20 \mathrm{mg} / \mathrm{kg} /$ día. Aunque su mecanismo de acción no se conoce a ciencia cierta, implica probablemente la inhibición de la captación de bilis por los hepatocitos y la inducción de enzimas microsómicas. El fenobarbital y la colestiramina son menos eficaces y poseen más efectos secundarios.

Deben efectuarse vacunaciones, especialmente si existe la probabilidad de un trasplante hepático futuro (fig. 6).

\section{Conclusión}

El diagnóstico precoz de la colestasis neonatal es importante si tenemos en cuenta la gravedad de la atresia biliar. El trasplante hepático ha cambiado espectacularmente el pronóstico tanto de la atresia biliar como de una gran cantidad de otras enfermedades colestáticas en niños. El progreso terapéutico en patología pediátrica está vinculado esencialmente al trasplante hepático, que proporciona una buena probabilidad de supervivencia y una buena calidad de vida. Los resultados a largo plazo del trasplante son favorables, con una tasa de supervivencia en torno al $90 \%$ al cabo de 5 años del trasplante. Sin embargo, se trata de una intervención muy dificultosa, con muchas complicaciones quirúrgicas y médicas, como insuficiencia renal y enfermedad linfoproliferativa postrasplante. Se han desarrollado tratamientos médicos específicos en algunos trastornos, como el tratamiento con NTBC en la tirosinemia de tipo I y con ácido cólico en los trastornos sintéticos de los ácidos biliares, tratamientos que permiten evitar el trasplante en la mayoría de los casos. Exceptuando estos dos casos concretos, el único tratamiento médico posible para la colestasis es un tratamiento inespecífico con ácido ursodesoxicólico. Los progresos recientes en el diagnóstico genético de las enfermedades colestáticas podrían permitir una mejor comprensión de los mecanismos fisiopatológicos y el desarrollo de nuevas estrategias terapéuticas como el trasplante de hepatocitos o la terapia génica. 


\section{Bibliografía}

1 Bernard O: Early diagnosis of neonatal cholestatic jaundice. Arch Pediatr 1998;5:10311035.

-2 Setchell D, Dumaswala R, Colombo C, Ronchi M: Hepatic bile acid metabolism during early development revealed from the analysis of human fetal gallbladder bile. J Biol Chem 1988;263:16637-16644.

-3 Jacquemin E: Development and physiopathology of biliary secretion. Arch Fr Pediatr 1992;49:741-748.

$\checkmark 4$ Chardot C, Carton M, Spire-Bendelac N, et al: Epidemiology of biliary atresia in France: a national study. J Hepatol 1999;31:10061013.

5 Chardot C, Carton M, Spire-Bendelac N, et al: Prognosis of biliary atresia in the era of liver transplantation: French national study from 1986 to 1996. Hepatology 1999;30:606611.

6 Debray D, Parient D, Urvoas E, et al: Sclerosing cholangitis in children. J Pediatr 1994;12: 49-56.

$\checkmark 7$ Amedee-Manesme O, Bernard O, Brunelle F, et al: Sclerosing cholangitis with neonatal onset. J Pediatr 1987;111:225-229.

$>8$ Hadj-Rabia S, Baala L, Vabres P, et al: Claudin-1 gene mutations in neonatal sclerosing cholangitis associated with ichthyosis: a tight junction disease. Gastroenterology 2004;127:1386-1390.

$\checkmark 9$ Feldmeyer L, Huber M, Fellmann F, et al: Confirmation of the origin of the NISCH syndrome. Hum Mutat 2006;27:408-410.

10 Krawinkel MB: Parenteral nutrition-associated cholestasis - what do we know, what can we do? Eur J Pediatr Surg 2004; 14:230-234.

$>11$ Lang C, Meier Y, Stieger B, et al: Mutations and polymorphisms in the bile salt export pump and the multidrug resistance protein 3 associated with drug-induced liver injury. Pharmacogenet Genomics 2007;17:47-60.

12 Herzog D, Chessex P, Martin S, Alvarez F: Transcient cholestasis in new born infants with perinatal asphyxia. Can J Gastroenterol 2003;17:179-182.
13 Vajro P, Amelio A, Stagni A, et al: Cholestasis in new born infants with perinatal asphyxia. Acta Paediatr 1997;86:895-898.

14 Lykavieris P, Hadchouel M, Chardot C, Bernard O: Outcome of liver disease with Alagille syndrome: a study of 163 patients. Gut 2001;49:431-435.

$\checkmark 15$ Crosnier C, Driancourt C, Raynaud N, et al: Mutations in JAGGED1 gene are predominantly sporadic in Alagille syndrome. Gastroenterology 1999;116:1141-1148.

16 Teckman JH: Alpha1-antitrypsine deficiency in childhood. Semin Liver Dis 2007;27: 274-281.

$>17$ Regev A, Guaqueta C, Molina EG, et al: Does the heterozygous state of alpha1-antitrypsine deficiency have a role in chronic liver diseases? Interim results of a large case-control study. J Pediatr Gastroenterol Nutr 2006; 43:S30-S35.

18 Hinds R, Hadchouel A, Shanmugham NP, et al: Variable involvement in siblings with PiZZ alpha-1-antitrypsine deficiency related liver disease. J Pediatr Gastroenterol Nutr 2006;43:136-138.

19 Lykavieris P, Bernard O, Hadchouel M: Neonatal cholestasis in childhood. Arch Dis Child 1996;75:67-70.

-20 Englert C, Grabhorn E, Richter A, et al: Liver transplantation in children with progressive familial intrahepatic cholestasis. Transplantation 2007;84:1361-1363.

21 Bustorff-Silva J, Sbraggia Neto L, Olimpio H, et al: Partial internal biliary diversion through a cholecystojejunocolonic anastomosis - a novel surgical approach for patients with progressive familial intrahepatic cholestasis: a preliminary report. J Pediatr Surg 2007;42: 1337-1340.

22 de Vree JM, Jacquemin E, Sturm E, et al: Mutation in the MDR3 gene cause progressive familial intrahepatic cholestasis. Proc Natl Acad Sci USA 1998;95:282-287.

23 Jung C, Driancourt C, Baussan C, et al: Prenatal molecular diagnosis of inherited cholestatis diseases. J Pediatr Gastroenterol Nutr 2007;44:453-458.

24 Russell DW, Setchell KDR: Bile acid biosynthesis. Biochemistry 1992;31:4737-4739.
25 Cheng JB, Jacquemin E, Gerhardt M, et al: Molecular genetics of 3beta-hydroxy-delta5C27 steroid oxidoreductase deficiency in 16 patients with loss of bile acid synthesis and liver disease. J Clin Endocrinol Metab 2003; 88:1833-1841.

26 Setchell KD, Heubi JE: Defects in bile acid biosynthesis - diagnosis and treatment. J Pediatr Gastroenterol Nutr 2006;43:17-22.

27 Yerushalmi B, Sokol RJ, Narkewicz MR, et al: Niemann-Pick disease type $\mathrm{C}$ in neonatal cholestasis at a North American center. J Pediatr Gastroenterol Nutr 2002;35:44-50.

$>28$ Lee WS, Sokol RJ: Liver disease in mitochondrial disorders. Semin Liver Dis 2007;27: 259-273.

29 Lee WS, Sokol RJ: Mitochondrial hepatopathies: advance in genetics and pathogenesis. Hepatology 2007;45:1555-1565.

30 Bosch AM: Classical galactosemia revisited. J Inherit Metab Dis 2006;29:516-525.

-31 McKiernan PJ: Nitisinone in the treatment of hereditary tyrosinemia type I. Drugs 2006; 66:743-750

32 Van Spronsen FJ, Bijleveld CM, Van Maldegem BT, Wijburg FA: Hepatocellular carcinoma in hereditary tyrosinemia type I despite 2-(2 nitro-4-3 trifluoro-methylbenzoyl)-1, 3-cyclohexanedione treatment. J Pediatr Gastroenterol Nutr 2005;40:90-93.

33 Depreter M, Espeel M, Roels F: Human peroxisomal disorder. Microsc Res Tech 2003; 61:203-223.

34 de Lonlay P, Seta N, Barrot S, et al: A broad spectrum of clinical presentations in congenital disorders of glycosylation I: a series of 26 cases. J Med Genet 2001;38:14-19.

$>35$ Jacquemin E, Lykavieris P, Chaoui N, et al: Transient neonatal cholestasis: origin and outcome. J Pediatr 1998;133:563-567.

36 Hermeziu B, Sanlaville D, Girard M, et al: Heterozygous bile salt export pump deficiency: a possible genetic predisposition to transient neonatal cholestasis. J Pediatr Gastroenterol Nutr 2006;42:114-116. 